\title{
Proportion of children with cancer that have an indication for genetic counseling and testing based on the cancer type irrespective of other features
}

\author{
Thi Minh Kha Nguyen ${ }^{1} \cdot$ Astrid Behnert ${ }^{1} \cdot$ Torsten Pietsch $^{2} \cdot$ Christian Vokuhl $^{3} \cdot$ Christian Peter Kratz $^{1}$ (i)
}

Received: 12 November 2020 / Accepted: 9 February 2021 / Published online: 26 February 2021

(c) The Author(s) 2021

\begin{abstract}
In children with cancer, specific clinical features such as physical anomalies, occurrence of cancer in young relatives, specific cancer histologies, and unique mutation/methylation signatures may indicate the presence of an underlying cancer predisposition syndrome (CPS). The proportion of children with a cancer type suggesting a CPS among all children with cancer is unknown. To determine the proportion of children with cancer types suggesting an underlying CPS among children with cancer. We evaluated the number of children with cancer types strongly associated with CPS diagnosed in Germany between 2007 and 2016. Data were obtained from various sources including two national pediatric pathology reference laboratories for brain and solid tumors, respectively, various childhood cancer trial offices as well as the German Childhood Cancer Registry. Among 21,127 children diagnosed with cancer between 2007 and 2016, 2554 (12.1\%) had a cancer type strongly associated with a CPS. The most common diagnoses were myelodysplastic syndrome and juvenile myelomonocytic leukemia, retinoblastoma, malignant peripheral nerve sheath tumor, infantile myofibromatosis, medulloblastoma ${ }^{\mathrm{SHH}}$, rhabdoid tumor as well as atypical teratoid/rhabdoid tumor. Based on cancer type only, $12.1 \%$ of all children with cancer have an indication for a genetic evaluation. Pediatric oncology patients require access to genetic counselling and testing.
\end{abstract}

Keywords Cancer predisposition syndromes $\cdot$ Pathology $\cdot$ Childhood cancer

\section{Introduction}

Cancer predisposition syndromes (CPS) are a well-established cause of childhood cancer [1-4]. Several clinical features may prompt a physician to consider an underlying CPS in a child with cancer. These include physical anomalies, positive cancer (family) history, and cancer type as well as somatic molecular features [4-7]. While there are cancer types rarely associated with a CPS, such as acute lymphoblastic leukemia (ALL), germ cell tumors, and

Christian Peter Kratz

kratz.christian@mh-hannover.de

1 Pediatric Hematology and Oncology, Hannover Medical School, Carl-Neuberg-Str. 1, 30625 Hannover, Germany

2 Department of Neuropathology, Institute of Neuropathology, Brain Tumor Reference Center, University of Bonn, Bonn, Germany

3 Section of Pediatric Pathology, Institute of Pathology, University Hospital Bonn, Bonn, Germany neuroblastoma, there are other cancer types that, by itself, always suggest the presence of an underlying CPS. Examples include pleuropulmonary blastoma strongly associated with DICERl germline variants or retinoblastoma strongly associated with pathogenic variants in $R B 1$. Here, we evaluated the proportion of children with cancer types strongly associated with CPS diagnosed within a ten-year, 2007-2016, period in Germany. Addressing this question is important in order to better estimate genetic counseling needs in the field of pediatric oncology.

\section{Methods}

Cancer types with an indication for genetic counseling and testing were recently reviewed and incorporated in a CPSquestionnaire currently used in Germany to identify patients with an indication for a genetic evaluation (see supplement to Ref. [6]). The list of cancer types falling into this category was created by the cancer predisposition working group of the German Society for Pediatric Oncology and 
Hematology and was based on recommendations from various cancer trial groups. The list shares large overlaps with a similar list generated independently by Postema et al. [4] who created a list of cancer types that warrant a genetic evaluation because at least $5 \%$ of cases are associated with a CPS. The number of patients with the following cancer types diagnosed in Germany between January 1st 2007 and December 31st 2016 were retrieved from the pediatric cancer pathology reference laboratories of the German Society of Oncology and Hematology (GPOH) in Bonn where generally all cases of childhood tumors are reviewed centrally: adrenocortical adenoma, adrenocortical carcinoma, atypical teratoid/rhabdoid tumor, botryoid rhabdomyosarcoma of the genitourinary tract, choroid plexus carcinoma, colorectal carcinoma, cystic nephroma, fetal rhabdomyoma, gastrointestinal stromal tumor, giant cell glioblastoma, gonadoblastoma, hemangioblastoma, hepatocellular carcinoma, infantile myofibromatosis, large cell calcifying Sertoli-cell-tumor, malignant peripheral nerve sheath tumor, medullary renal cell carcinoma, medullary thyroid carcinoma, medulloepithelioma, melanoma, meningioma, myxoma, neuroendocrine tumor (excluding carcinoid of the appendix), optic pathway glioma, parathyroid adenoma, pheochromocytoma, pilocytic astrocytoma with signs of neurofibromatosis type 1 , pineoblastoma, pituitary adenoma/tumor, pleuropulmonary blastoma, renal cell carcinoma, rhabdoid-tumor, anaplastic rhabdomyosarcoma, schwannoma, Sertoli-Leydig-celltumor, small cell carcinoma of the ovary hypercalcemic type, squamous cell carcinoma, subependymal giant cell astrocytoma, thyroid carcinoma (follicular), thyroid carcinoma (papillary), thyroid carcinoma (unclassified). In addition, the number of patients with the following cancer types diagnosed within the same 10-year period were retrieved from the annual report of the German Childhood Cancer Registry (GCCR) [8]: myelodysplastic syndrome/juvenile myelomonocytic leukemia, and retinoblastoma.

The number of patients diagnosed in Germany within the same 10-year period with the following entities were calculated based on numbers provided in the annual report of the GCCR [8] and the published percentage of cases with the defining features [9-12]: medulloblastoma ${ }^{\mathrm{SHH}}$, medulloblastoma ${ }^{\text {WNT }}$ CTNNB1 wildtype, hepatoblastoma CTNNB 1 wildtype and acute myeloid leukemia (AML) with monosomy $7 /$ aberration 7q. Finally, the number of patients diagnosed with hypodiploid ALL was provided by the ALL BFM and COALL trial groups (C.P.K. personal communication).

Beginning in 2009, the GCCR extended the upper age cut-off from 15 years to 18 years. According to the GCCR's archived annual reports, 16,902 patients under the age of 18 years were diagnosed between years 2009 and 2016 (on average, 2112 patients per year). Based on this number, we estimate that 21,127 patients under the age of 18 years were diagnosed with cancer between January 1st 2007 and December 31 st 2016 . The study was approved by the ethical review board at Hannover Medical School.

\section{Results}

Among the 21,127 patients diagnosed with cancer prior to age 18 years in Germany between January 1st 2007 and December 31st 2016, 2554 children (12.1\%) had a cancer type highly suggesting the presence of an underlying CPS. The most common diagnoses were myelodysplastic syndrome, juvenile myelomonocytic leukemia, retinoblastoma, malignant peripheral nerve sheath tumor, infantile myofibromatosis, medulloblastoma ${ }^{\mathrm{SHH}}$, rhabdoid tumor and atypical teratoid/rhabdoid tumor. For all the remaining entities there were less than 10 patients per year diagnosed in Germany (Fig. 1).

\section{Discussion}

Approximately $10 \%$ of children with cancer have a CPS [1-4]. Therefore, a CPS should be, at least, considered, in every child with cancer. The optimal approach to diagnose children with a CPS among children with cancer is currently being investigated by several groups. One strategy is to clinically identify individual patients who have a particular high probability of an underlying CPS and to offer genetic evaluation to these individuals. We and others have established simple tools in order to help physicians to identify patients with an indication for genetic counseling and testing [4-7]. In addition to family history and physical anomalies, the cancer type (including somatic signatures) may help to identify patients with a high probability of an underlying CPS. The cancer types falling into this category are constantly growing and evolving [13]. Here, we determined the proportion of children with cancer types highly suggestive of an underlying CPS and found that $14 \%$ of children with cancer fall into this category.

The study has several limitations: (1) The study design did not allow us to determine the percentage of patients who had an underlying CPS; It is likely that many patients were never genetically evaluated because in the past patients were not studied systematically; (2) We cannot rule out that we missed patients because our main identifying sources were two reference laboratories. It is possible that not all patients with relevant cancer types were reviewed by these reference laboratories. Nevertheless, the use of pathology reference laboratories is a common practice in Germany and generally, most pediatric cases are reviewed. We cannot rule out, however, that all pathologies from children with "adult" tumor types 
Fig. 1 Number of children with various cancer types highly associated with cancer predisposition syndromes diagnosed in Germany within a 10 -year period, 2007-2016 myel odysplastic syndrome / juvenile myelomonocytic leukemia retinoblastoma

infantile myo fibromatosis malignant peripheral nerve sheath tumor

medulloblastoma $\mathrm{SHH}$ atypical teratoid/rhabdoid tumor

rhabdoid-tumor

meningeoma botryoid rhabdomyosarcoma of the genitourinary tract

hepatocellular carcinoma hypodiploid acute lymphoblastic leukemia renal cell carcino ma neuroendocrine tumor pilocytic aststo cytoma with signs of NF1 adrenocortical carcin oma schwannoma choroid plexus carcinoma

pheochromocytoma

adrenocortical aden oma pilocytic astostoma chiasma thyroid carcinoma (papillary) Sertoli-Leydig-cell-tumor pineoblastoma large cell calcifying Sertoli-cell-tumor subependymal giant cell astrocytoma pleuropulmonary blastoma melanoma

giant cell glioblastoma optic pathway glioma hepatoblstoma CTNNB1 wildtype

$$
\text { cystic nephroma }
$$

AML with monosomy $7 /$ aberration $7 q$

myxoma

pituitary adenoma / tumor squamous cell carcinoma

hemangioblastoma

gonadoblastoma

fetal rhabdomyoma

gastrointestinal stromal tumor

medullary thyroid carcinoma

medulloep ithelioma

medulloblastoma WNT CTNNB1 wild type ।

small cell carcin. of the ovary hypercalcemic type I

rhabdomyosarcoma anaplastic ।

colorectal carcin oma

thyroid carconima (unclassified)

thyroid carcinoma (follicular)

parathyroid adenoma

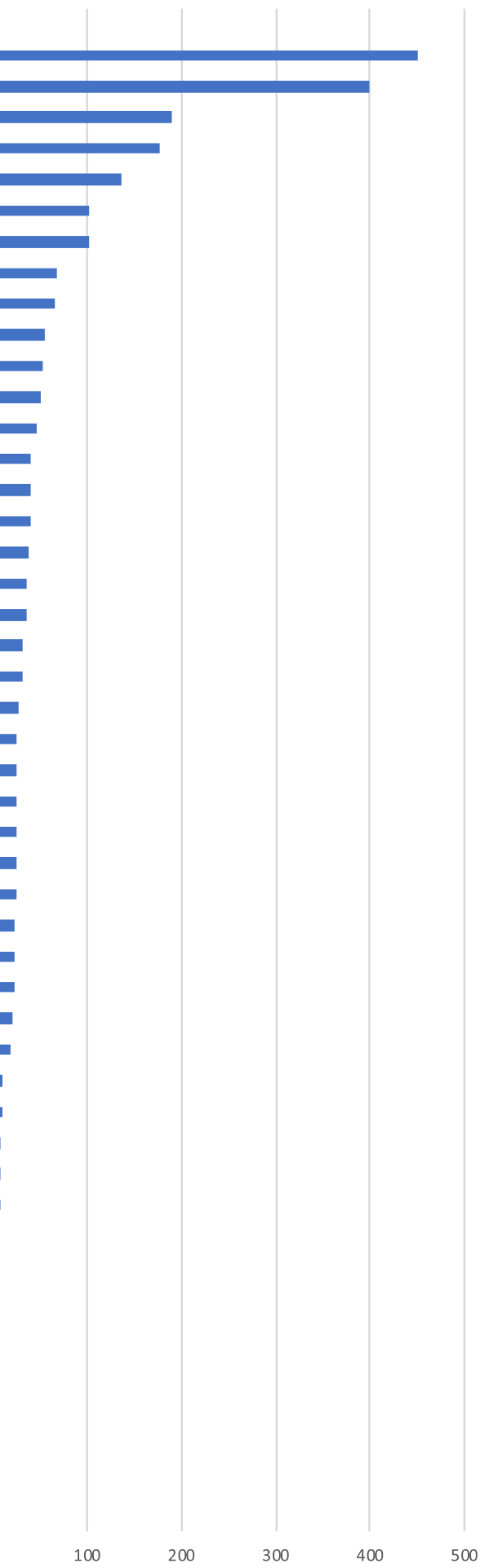

(e.g. colorectal carcinoma) were reviewed by one of the pediatric reference laboratories that served as our main source; (3) not all tumors are biopsied (e.g., optic pathway glioma in patients with neufibromatosis type 1 are not always biopsied) potentially leading to an underestimation in this study. (4) The cancer types associated with CPS are constantly evolving. For example, after our data collection was completed, TRIM28 pathogenic variants were identified in epithelial nephroblastoma [14-17]. Thus, our list of cancer types is incomplete, leading to an underestimation 
of the determined proportion; (5) Several cancer types, especially brain tumors, are now subdivided into molecular subgroups. Only recently it has been shown that some of these subgroups are often associated with a CPS and, therefore, these data were not available for the entire 10 -year period. We were able to compensate this problem by providing estimates in this situation (see "Methods" section).

In conclusion, $12.1 \%$ of children with cancer have a cancer type strongly associated with a CPS. In order to increase the chance that affected families are being offered counseling and testing, reference pathology reports on these entities should state that a genetic evaluation is recommended. We also recommend that pediatric oncologists use one of the available screening tools [4-7]-some of which use decisional algorithms [7]-to identify patients with a high likelihood of an underlying CPS.

Author contributions The study was concepted by CPK, TMKN, and AB Data and material of the study was generated by CV and TP Data collection was performed by TMKN, interpretation and analysis were conducted by TMKN, with the support of CPK and $\mathrm{AB}$. TMKN wrote the manuscript with the support of $\mathrm{CPK}$ and $\mathrm{AB}$. The paper was edited by all authors. All authors have read and approved the final manuscript.

Funding Open Access funding enabled and organized by Projekt DEAL. CPK has been supported by the Deutsche Kinderkrebsstiftung (DKS2017.02), and BMBF ADDRess (01GM1909A).

Data availability Raw data and material and processed data are held within the Department of Pediatric Hematology and Oncology at Hannover Medical School.

\section{Compliance with ethical standards}

Conflict of interest The authors indicate no potential conflicts of interest.

Ethics approval The study was approved by the ethical review board at Hannover Medical School.

Consent to participate and for publication A consent was not necessary in this retrospective analysis.

Open Access This article is licensed under a Creative Commons Attribution 4.0 International License, which permits use, sharing, adaptation, distribution and reproduction in any medium or format, as long as you give appropriate credit to the original author(s) and the source, provide a link to the Creative Commons licence, and indicate if changes were made. The images or other third party material in this article are included in the article's Creative Commons licence, unless indicated otherwise in a credit line to the material. If material is not included in the article's Creative Commons licence and your intended use is not permitted by statutory regulation or exceeds the permitted use, you will need to obtain permission directly from the copyright holder. To view a copy of this licence, visit http://creativecommons.org/licenses/by/4.0/.

\section{References}

1. Sylvester DE, Chen Y, Jamieson RV, Dalla-Pozza L, Byrne JA (2018) Investigation of clinically relevant germline variants detected by next-generation sequencing in patients with childhood cancer: a review of the literature. J Med Genet 55(12):785-793. https://doi.org/10.1136/jmedgenet-2018-10548 8

2. Zhang J, Walsh MF, Wu G et al (2015) Germline mutations in predisposition genes in pediatric cancer. N Engl J Med 373(24):2336-2346. https://doi.org/10.1056/NEJMoa1508054

3. Grobner SN, Worst BC, Weischenfeldt J et al (2018) The landscape of genomic alterations across childhood cancers. Nature 555(7696):321-327. https://doi.org/10.1038/nature25480

4. Postema FAM, Hopman SMJ, Aalfs CM et al (2017) Childhood tumours with a high probability of being part of a tumour predisposition syndrome; reason for referral for genetic consultation. Eur J Cancer 80:48-54. https://doi.org/10.1016/j.ejca.2017.04.021

5. Jongmans MC, Loeffen JL, Waanders E et al (2016) Recognition of genetic predisposition in pediatric cancer patients: an easyto-use selection tool. Eur J Med Genet 59(3):116-125. https:// doi.org/10.1016/j.ejmg.2016.01.008

6. Ripperger T, Bielack SS, Borkhardt A et al (2017) Childhood cancer predisposition syndromes-a concise review and recommendations by the Cancer Predisposition Working Group of the Society for Pediatric Oncology and Hematology. Am J Med Genet A 173(4):1017-1037. https://doi.org/10.1002/ajmg.a.38142

7. Goudie C, Cullinan N, Villani A et al (2018) Retrospective evaluation of a decision-support algorithm (MIPOGG) for genetic referrals for children with neuroblastic tumors. Pediatr Blood Cancer 65(12):e27390. https://doi.org/10.1002/pbc.27390

8. Kaatsch P, Grabow D, Spix C (2018) German Childhood Cancer Registry - Annual Report 2017 (1980-2016). Institute of Medical Biostatistics, Epidemiology and Informatics (IMBEI) at the University Medical Center of the Johannes Gutenberg University Mainz, 2018

9. Waszak SM, Northcott PA, Buchhalter I et al (2018) Spectrum and prevalence of genetic predisposition in medulloblastoma: a retrospective genetic study and prospective validation in a clinical trial cohort. Lancet Oncol 19(6):785-798. https://doi. org/10.1016/S1470-2045(18)30242-0

10. Northcott PA, Robinson GW, Kratz CP et al (2019) Medulloblastoma. Nat Rev Dis Primers 5(1):11. https://doi.org/10.1038/ s41572-019-0063-6

11. Rasche M, von Neuhoff C, Dworzak M et al (2017) Genotype-outcome correlations in pediatric AML: the impact of a monosomal karyotype in trial AML-BFM 2004. Leukemia 31(12):2807-2814. https://doi.org/10.1038/leu.2017.121

12. Aretz S, Koch A, Uhlhaas S et al (2006) Should children at risk for familial adenomatous polyposis be screened for hepatoblastoma and children with apparently sporadic hepatoblastoma be screened for APC germline mutations? Pediatr Blood Cancer 47(6):811-818. https://doi.org/10.1002/pbc.20698

13. Apellaniz-Ruiz M, Segni M, Kettwig M et al (2019) Mesenchymal Hamartoma of the Liver and DICER1 Syndrome. N Engl J Med 380(19):1834-1842. https://doi.org/10.1056/NEJMoa1812169

14. Mahamdallie S, Yost S, Poyastro-Pearson E et al (2019) Identification of new Wilms tumour predisposition genes: an exome sequencing study. Lancet Child Adolesc Health 3(5):322-331. https://doi.org/10.1016/S2352-4642(19)30018-5

15. Diets IJ, Hoyer J, Ekici AB et al (2019) TRIM28 haploinsufficiency predisposes to Wilms tumor. Int J Cancer 145(4):941951. https://doi.org/10.1002/ijc.32167

16. Halliday BJ, Fukuzawa R, Markie DM et al (2018) Germline mutations and somatic inactivation of TRIM28 in Wilms 
tumour. PLoS Genet 14(6):e1007399. https://doi.org/10.1371/ journal.pgen.1007399

17. Armstrong AE, Gadd S, Huff V, Gerhard DS, Dome JS, Perlman EJ (2018) A unique subset of low-risk Wilms tumors is characterized by loss of function of TRIM28 (KAP1), a gene critical in early renal development: a Children's Oncology Group study. PLoS ONE 13(12):e0208936. https://doi.org/10.1371/journ al.pone.0208936
Publisher's note Springer Nature remains neutral with regard to jurisdictional claims in published maps and institutional affiliations. 